\title{
An investigation on vibration-based damage detection in circular
}

\author{
plates \\ I.Trendafoilova ${ }^{1}$, D.G Gorman ${ }^{1}$ and E. Manoach ${ }^{2}$ \\ $\underline{\text { Irina.Trendafilova@strath.ac.uk }}$ \\ Daniel.Gorman@strath.ac.uk \\ e.manoach@info.imbm.bas.bg
}

\begin{abstract}
This study aims at the development of vibration-based health monitoring (VHM) methodology for thin circular plates. The possibility of using the first several natural frequencies of a circular plate for damage detection purposes is investigated first. The study then suggests a damage detection method, which considers a vibrating plate as a dynamic system and uses its time domain response represented in a new phase (state) space to extract damage sensitive characteristics. The paper introduces the idea of using large amplitude vibrations and nonlinear time series analysis for damage detection purposes. The suggested damage detection approach explores the possibility to use certain characteristics of the distribution of phase space points on the attractor of the system. It studies the histograms of this distribution and attempts to extract damage sensitive features. Three damage features are suggested and they are shown to detect damage at a rather low level using a finite element model of the plate. The method suggested is rather generic and permits development and application to more complex structures and real data.
\end{abstract}

Keywords: vibration-based health monitoring, damage detection, structural vibrations, nonlinear dynamics, state space.

1 Department of Mechanical Engineering, James Weir Building, University of Strathclyde, Glasgow G1 1XJ, UK.

2 Institute of Mechanics, Bulgarian Academy of Sciences, Sofia, Bulgaria 


\section{Introduction.}

Damage generated within a structure usually leads to changes in the physical properties of the structure which in turn result in changes in its vibration response. Vibration health monitoring (VHM) is a method, which uses the changes in the vibration response of structure for the purposes of fault (damage) diagnosis. VHM is generally accepted as a method for fault diagnosis, but its practical application to a number of structures still poses problems and challenges. This is why establishing the applicability of VHM to a certain type of structures is already an important step towards the eventual development of a practically applied procedure. The applicability as well as the concrete development of a VHM method is very much case dependent and is normally aimed at a certain structure or type of structures. The changes in certain vibration characteristics are also case dependent and they depend on the structure, the material, the support conditions, as well as the environmental factors. As far as damage is in most cases a local phenomenon, its influence on the global dynamic characteristics of the structure, such as lower natural frequencies, might be weak for some structures. But this is not a rule and the sensitivity of the lower natural frequencies to damage should be established for a certain structural type. Even in cases where the natural frequencies turn out to be damage sensitive, special care should be taken about other factors influencing these frequencies. In general it is assumed that the vibrating structure does not interact with the environment, and that there is no influence of any surrounding temperature or fluid or that this influence can be neglected. However the influence of the environmental conditions on the lower natural frequencies might represent an additional problem when using these natural frequencies as damage features [1]. In a recent paper we developed a procedure, which can take into account the influence of an acoustic 
cavity around the circular plate on its lower natural frequencies and eventually exclude this influence [2]. Thus the pure structural natural frequencies can be extracted from the measured ones, which are subjected to a certain fluid-structure interaction. This procedure allows to use the lower natural frequencies of a certain structure as damage features in case they show enough sensitivity to certain defects.

This paper aims at the development of a viable VHM method for thin circular plates. Accordingly we first examine the sensitivity of the lower natural frequencies to certain types of damage and attempt to establish the type and size of defects which may be detectable using these frequencies. As it is expected, the sensitivity of the first several natural frequencies to damage is rather limited. It is known that small damage is easier to detect examining large amplitude vibrations, which are much more affected by the nonlinearities in a structure. The next task of the paper is therefore to introduce and develop an alternative damage detection approach. It uses large amplitude vibrations and is based on the time domain structural vibration response This approach suggests the use of nonlinear time series analysis to account for any nonlinearities, including damage, which become more important when large amplitude vibrations are present. The time-domain signal analysis was found to have a number of advantages compared to other plausible damage detection procedures e.g. mode shape-based methods and methods using FE model updating. The use and the comparison of the structural mode shapes is not that straightforward and might involve the estimation of curvatures which will introduce further inaccuracies [19]. FE model updating methods might give a false alarm due to discrepancies between the modelled and the measured structural vibration response. The use of the pure time domain measured structural response does not involve any assumptions for model and/or linearity and is not expected to suffer any of the above problems. The idea of 
using the time domain structural response and nonlinear time series analysis for damage detection is not new. It has been explored by authors of this study $[3,4]$ as well as by others [5-8]. The novelty of the suggested approach is in the utilisation of the distribution of phase space points and their histogram to establish certain damage sensitive characteristics. The paper also suggests a simple and straightforward method to estimate these characteristics from measured data. Since these are statistical characteristics and they characterise the distribution of phase space points on the attractor, they are expected to be somewhat robust to noise and environmental changes. Such circumstances may be considered to partly relieve the necessity to take into account the influence of noise and environmental conditions on the dynamic response of the structure.

\section{The plate, the model and the considered damage.}

This study is based on finite element modelling. A thin circular aluminium plate of a radius of $500 \mathrm{~mm}$ and thickness of $5 \mathrm{~mm}$ is modelled using 3D elements. The geometrically non-linear version of the so called Raisner-Mindlin plate theory was used to describe the dynamic plate behaviour and to account for large amplitude vibrations. This theory takes into account the influence of the shear stress and angular rotations on the plate behaviour and gives more adequate results than the classical one especially in the case of nonlinear and large amplitude vibrations. The RaisnerMindlin theory and its FE modelling application are considered in detail in e.g. $[16,17]$ The same theory was used by the authors to model large amplitude vibrations in other structures [18]. In order to excite large amplitude vibrations the plate is subjected to harmonic excitation with amplitudes $1000 \mathrm{kN}$ and $2000 \mathrm{kN}$ close to the first and the second natural frequencies of the plate $\left(\omega_{1}=1250 \mathrm{kN}, \omega_{2}=2100 \mathrm{kN}\right)$. The following material characteristics were assumed for aluminium: Young's modulus E= 
$7.10^{10} \mathrm{~N} / \mathrm{m}^{2}$, Poison ratio $v=0.34$, density $\rho=2778 \mathrm{~kg} / \mathrm{m}^{3}$. Two types of damage in the plate are considered: 1) concentrated damage in the form of a crack and 2) distributed damage, which might be a result of corrosion or crack distribution. Cracks were modelled by disbanding a number of elements in the plate. The size of the crack was modified by changing the number of disbanded elements. Distributed damage was modelled by stiffness reduction in a certain region of the plate. Several levels of distributed damage were considered by different stiffness reductions levels. The loading was applied in the middle of the plate and the vibration response was calculated for 60 equidistantly distributed points on 12 radiuses of the plate at an angle of $30^{\circ}$ from each other, so that each 12 points form a circle with centre in the plate centre and with radiuses of $100,200, \ldots, 500 \mathrm{~mm}$. The plate was clamped at the periphery. The three damage scenarios considered are shown on Figure 1. The crack is assumed to start from the end of the plate and propagate towards its centre. The distributed damage is assumed to cover a small square with a $50 \mathrm{~mm}$ side. Two locations for such distributed damage are considered- central damage (case A) and end damage (case B). In case A the centre of the square is in the centre of the plate while in case B the square centre is $470 \mathrm{~mm}$ from the centre of the plate.

\section{The lower natural frequencies of the plate and damage detection.}

Different amplitudes of harmonic excitation close to the natural frequencies of the plate (e.g. $1000 \mathrm{~N} / \omega_{1}=1250 \mathrm{kN}, 2000 \mathrm{~N} / \omega_{2}=2100 \mathrm{kN}, 2600 \mathrm{kN} / \omega_{3}=2700 \mathrm{kN}, 5000$ $\mathrm{N} / \omega_{6}=4900 \mathrm{kN}$ ) were applied. It was found that the first natural frequencies of the plate are not notably affected by damage. The biggest changes were excited by the $1000 \mathrm{~N}$ amplitude excitation. The results for the relative changes in per cent in the first 
six natural frequencies of the plate (excited by $1000 \mathrm{~N}$ amplitude excitation) caused by a crack and by distributed damage are shown in Table 1. The higher frequencies (from the 7-th to the 10-th) were found to undergo even smaller changes than the first six ones in this particular case. This is the reason why they are not shown in the table.

The differences caused by other amplitude harmonic excitations are smaller than the ones given in Table 1.Other types of excitation (e.g. random) caused even smaller changes in the first several natural frequencies.

Small cracks are not likely to affect the first natural frequencies and this is the case with a lot of structures. In this particular case it was found that cracks, with length less than $20 \%$ of the plate radius $(100 \mathrm{~mm})$ cause a negligible reduction in the first 10 natural frequencies, which was calculated to be not larger that $1.2 \%$. But cracks with length equal to and longer than $100 \mathrm{~mm}$ caused more visible changes of about 5\% and more in some of the first 10 frequencies ( see Table 1). Small changes (e.g. less than 5\%) in the natural frequencies, can be caused by a number of factors including temperature changes, interaction with a fluid, or even just noise. In this study 5\% was used as a threshold value and it was assumed that changes of less than $5 \%$ will be considered insufficient for the purposes of damage detection. Accordingly it was assumed that the lower natural frequencies are not appropriate to detect thin cracks of length less than $20 \%$ of the plate radius.

Distributed damage up to a certain level incurred very small or no changes of lower natural frequencies. Stiffness reduction of $30 \%$ in a small square $(50 \mathrm{~mm} \times 50$ $\mathrm{mm})$ in the centre of the plate changed some of the natural frequencies with about $5 \%$. Stiffness reduction of about $40 \%$ was required for the same size square close to the end of the plate to get a similar drop in the natural frequencies. Table 1 shows the relative changes in per cent in the first 6 natural frequencies for the case of central 
defect (case A) and side defect (case B) for different stiffness reductions between $30 \%$ and $50 \%$. Smaller stiffness reductions (less than $30 \%$ for the central defect and less than $40 \%$ for the side defect) caused much smaller changes of less than $2.5 \%$ in the lower natural frequencies so it was assumed that the first 6 natural frequencies are not appropriate for detecting distributed damage at lower levels.

\section{The state-space approach and damage detection.}

The state (phase) space approach has been used for analysis of vibrating systems and for VHM purposes in several previous papers [3-5,8] and we shall introduce it very briefly here. It presents a way to study the time domain structural response. The measured structural response is normally represented by one dimensional vriables ( acceleration, displacement, velocities). But the structure considered is much more complicated and thus cannot be represented in a single dimension. In essence the state space approach finds a new multidimensional space, in which the dynamics of the structure can be fully reconstructed from its measured response [9]. It is assumed that the vibrating structure can be represented like any dynamic system by the following system of differential equations $[4,9]$ :

$$
\frac{d \mathbf{x}}{d t}=\mathbf{F}(\mathbf{x}(\mathbf{t}))
$$

But normally the original space $\mathbf{x}$ as well as the vector function $\mathbf{F}$ in equation (1) are not known. The state space approach is based on Taken's theorem, which asserts that the above system's dynamics can be recovered in a new space $\mathbf{y}$ made of time lags of a measured scalar time dependent system variable $s(n)=s(g(x(n))$. The new vectors $\mathbf{y}$

$$
\mathbf{y}(n)=[s(n), s(n+T), \ldots, s(n+(m-1) T)]
$$


composed simply of time lags of the observation which define the motion in an $\mathrm{m}$ dimensional Euclidean space. In particular it is shown that the evolution in time of the points $\mathbf{y}(n) \rightarrow \mathbf{y}(n+1)$ follows that of the unknown dynamics $\mathbf{x}(n) \rightarrow \mathbf{x}(n+1)$ [9]. $T$ and $m$ are properly chosen time delay and dimension respectively. Thus it is shown that if we are able to measure a single time-dependent quantity characterising our vibrating structure like e.g. its acceleration or displacement then one will be able to find a new multidimensional space constructed of time lags of the measured variable in which the dynamics of our structure can be fully reconstructed. The time lag and the dimension of the new state space are known as its embedding parameters. The proper choice of these parameters whilst out of the scope of this paper is explained in detail in e.g. $[10,11]$.

Once the new state space is found one needs to reconstruct the dynamics of the system in it. Since in most cases an explicit relation describing the dynamics of the system is not known, there are alternative characteristics in the state space which can be used. One way to characterise the dynamics of a structure in a state space is to use its dynamic invariants, which are closely related to its modal characteristics-natural frequencies and mode shapes [3,4,8,9]. A serious obstacle is the difficulty to determine the dynamic invariants from measured data $[4,10]$. An alternative way to characterise the dynamics of a structure in a state space is to study its attractor - the subset of the state space where all trajectories of a dynamic system converge $[9,10,11]$. The attractor, like any set of points, can be characterised by its statistical characteristics-distribution of the points, mean value, variance and higher statistical moments. These characteristics give information about the geometry of this set and its main properties. The ergodic theory [12] relates the dynamic invariants and the statistical characteristics of the attractor and permits the invariants to be viewed as 
invariant statistical quantities of the attractor. Since it is clear that most damage scenarios affect the modal characteristics of a structure then it can be argued and some of ours and other autors' research show that damage can affect the invariants of the motion of a vibrating structure and hence - the geometry of its state space $[3,4,5,7,8]$.

This piece of research concentrates on studying the distribution of points on the attractor and analysing the effect of damage on some of its statistical properties for the case of a circular plate. The novelty of the approach applied here is that it studies the joint two-dimensional histograms of the distribution of points and derives viable damage detection features on the basis of this study. Three global damage detection features are suggested, which can be used to detect damage in circular plates.

\section{Reconstructing the state space of vibrating system and a set of points on it.}

The measured quantity $s(n)$ of a vibrating system which can be used to reconstruct its state space is given in terms of either acceleration, velocity or displacement in a given point. The accelerations provide a quantity which is easy and straightforward to measure and for the purposes of this method we focus on the measured accelerations. A proper time lag $T$ between two consecutive measurements is found using the average mutual information [11]. A dimension $m=2$ is used here assuming that most of the statistical characteristics of the attractor will be preserved $[3,4]$. Suppose one measures a long enough acceleration record. It can be represented by an acceleration vector $\mathbf{a}$ as follows:

$$
\begin{aligned}
& \mathbf{a}=\left[a\left(t_{1}\right), a\left(t_{2}\right), \ldots, a\left(t_{n}\right)\right]^{T}=\left[a_{1}, a_{2}, \ldots, a_{n}\right]^{T} \\
& t_{i}=t_{i-1}+T \\
& i=1,2, \ldots, n
\end{aligned}
$$


where $a\left(t_{i}\right)$ are the measured accelerations in the time moments $t_{i}, i=1,2 \ldots, n, n$ is large enough, and the superscript $[. . .]^{T}$ stands for transpose. The normalised response of the structure $\mathbf{w}$ is used here (in order to make it independent on the excitation), which can be formed by dividing the acceleration response values to the maximum value of the excitation vector $[\mathbf{u}]^{T}$ :

$$
\mathbf{w}=\left\{w_{i}\right\}_{i=1}^{n}=\frac{a_{i}}{\max _{k}\left\{u_{k}\right\}}
$$

where $u_{k}, k=1,2, \ldots, 1$ make the excitation (force) vector, which is formed the same way as the vector $\mathbf{a}$ from Equation (4). The vectors $\mathbf{w}$ are used to obtain the new state space points $\mathbf{y}$. From a vector $\mathbf{w}$ one can obtain $n-1$ two dimensional state space points:

$$
\begin{aligned}
& y_{i}=\left[y_{i 1}, y_{i 2}\right]^{T}=\left[w_{i}, w_{i+1}\right]^{T}=\left[w\left(t_{i}\right), w\left(t_{i}+T\right)\right]^{T} \\
& i=1,2, \ldots, n-1
\end{aligned}
$$

The $\mathbf{y}$ vectors (6) made of the time lags of the normalised accelerations define the new phase space. A set of $N$ points $\mathbf{y}_{\mathrm{k}}, k=1,2, \ldots N$, is then randomly chosen on the response attractor and $N_{B}$ nearest neighbours are found for each point in the sense of Euclidean distance, $\quad \mathbf{y}_{q}^{i}, \quad i=1,2, \ldots, N, \quad q=1,2, \ldots N_{B}$. This set is denoted by $\mathbf{Y}_{n}=\left\{\left.\mathbf{y}_{q}^{k}\right|_{q=1,2, \ldots N_{B}} ^{k=1,2, \ldots N}\right\}$. This new set of $M, M=N . N_{B}$, two-dimensional state space points $\mathbf{Y}_{n}, n=1,2, \ldots, M$, is used to characterise the attractor of the vibrating plate in the y state space.

\section{The statistical distribution of phase space points and damage detection.}


In order to examine the distribution of the set of points $\mathbf{Y}_{n}$ obtained above it is useful to construct its histogram. The obtained set $\mathrm{Y}_{n}$ contains two-dimensional vectors $\mathbf{y}_{q}^{i}$, so we are interested in the joint (two dimensional) histogram of this set. The first thing one needs to confirm is if this joint distribution undergoes changes with damage. the joint histograms for the non-damaged case and for both damage cases, a crack and distributed damage, for different damage levels will be examined. All the results given below correspond to harmonic excitation with $1000 \mathrm{~N}$ amplitude.

We concentrate first on the problem for crack detection. The size of the crack was varied as explained above (\&2). We explored different locations and different sizes and it was found that a crack, which is less than $5 \%$ of the plate radius $(25 \mathrm{~mm})$ causes very small changes in the histogram of the points $Y_{i}$. The average relative Euclidean distance between the two histograms, which can be used to judge for the difference between them, is defined as follows:

$$
\bar{d}=\frac{1}{K} \sum_{\mathbf{j}=\mathbf{1}}^{K} \sqrt{\frac{\left(\mathbf{h}_{\mathbf{j}}-\mathbf{h}_{\mathbf{j}}^{\mathbf{d}}\right)^{T} \cdot\left(\mathbf{h}_{\mathbf{j}}-\mathbf{h}_{\mathbf{j}}^{\mathbf{d}}\right)}{\left(\mathbf{h}_{\mathbf{j}}\right)^{T} \cdot \mathbf{h}_{\mathbf{j}}}}
$$

where $\mathbf{h}_{\mathbf{j}}$ are the points on the histogram of the undamaged state and $\mathbf{h}_{\mathbf{j}}^{\mathbf{d}}$ are the points on the histogram of the damaged state and $K$ is the number of points on the histograms. The distance $\bar{d}$ represents the average relative change in the lengths of the vectors $\mathbf{h}_{\mathbf{j}}$ as a result of the introduced damage, which can be given in per cent similarly to the way we represented the change in natural frequencies. This distance was rather low, more precisely it was less than 0.01 ( $1 \%$ ) for the case of cracks, which are less than $5 \%$ of the plate radius. With the growth of the cracks this distance increases. Table 2 contains the distances $\bar{d}$ in per cent for the case of a horizontal 
crack (see Figure 1). It can be seen that this distance can be used to detect the presence of a crack.

Figure 2 a) and b) gives the histograms for the case of no damage as compared to the histograms for two crack sizes $30 \%$ and $10 \%$ cracks. The first conclusion by looking at the histograms is that they obviously change with the introduction and the growth of the crack. Another trend that can be observed from the histograms is the distributions becomes more "ordered" and approach the normal distribution with the introduction and the growth of a crack.

The next question is to find some characteristics that change with damage. We already introduced the average relative Euclidean distance between the joint histograms and this seems one possibility for a crack detection feature. Another feature, that can be perceived by looking at the joint histograms, is the spread. It can be seen that it goes down with damage - the histogram that corresponds to the nondamaged case has a much bigger spread than the ones corresponding to the damaged states. The same can be observed for the symmetry of the distributions- the less damage the less symmetric the distribution becomes. So the non-damaged state seems to possess the least symmetric distribution, while the histogram corresponding to the biggest crack is the most symmetric one. Thus the most "disordered" state corresponds to the non-damaged case and introducing damage makes the dynamics more ordered and the most symmetric geometry with the least spread corresponds to biggest damage. These conclusions are in agreement with previous observations which conclude that the dynamics of the structure characterised by the set of points $\mathbf{Y}_{n}$ becomes more "ordered" with damage [3-7].

The two statistical characteristics associated with the spread and the symmetry of the distribution are the variance and the skewness, which are given by the second 
and the third moments of the distribution. These characteristics can be defined by the following scalar variables for a multidimensional distribution :

$$
\begin{aligned}
& \sigma=\frac{1}{M} \sum_{i=1}^{M}\left(\mathbf{Y}_{\mathbf{i}}-\overline{\mathbf{Y}}\right)^{\prime}\left(\mathbf{Y}_{\mathbf{i}}-\overline{\mathbf{Y}}\right) \\
& s=\frac{1}{M^{2}} \sum_{i=1}^{M} \sum_{j=1}^{M}\left[\left(\mathbf{Y}_{\mathbf{i}}-\overline{\mathbf{Y}}\right)^{\prime} \mathbf{S}^{-1}\left(\mathbf{Y}_{\mathbf{j}}-\overline{\mathbf{Y}}\right)\right]^{3}
\end{aligned}
$$

where $M$ is the number of points $\mathbf{Y}_{\mathbf{i}}, \mathbf{i}=1, \ldots, M, \overline{\mathbf{Y}}$ is the sample mean vector and $\mathbf{S}$ is the sample covariance matrix. The above quantities were introduced by Mardia [13] and we shall call them sample multivariate variance $\sigma$ and skewness $s$. These quantities give an average overall estimate of the variance and skewness of the distribution of points $\mathbf{Y}$. In order to perceive the changes that the parameters $\sigma$ and $s$ undergo with damage, instead of using the values for $\sigma$ and $s$ one can introduce relative changes compared to the non damaged case (similar to the average relative distance between the histograms introduced by Equation (7) ). They are introduced as follows:

$$
\begin{aligned}
& \bar{\sigma}=\frac{\left(\sigma_{n}-\sigma\right)}{\sigma_{n}} \\
& \bar{s}=\frac{\sqrt{\left(s_{n}-s\right)^{2}}}{s_{n}}
\end{aligned}
$$

where $\sigma_{n}$ and $s_{n}$ are the values of the sample variance and skewness for the non damaged case and $\sigma$ and $s$ correspond to the current (possibly damaged) state. We have taken the absolute value of the skewness change since $s_{n}$ and $s$ can be positive or negative and we are interested in the overall change of the skewness. The values of the above changes in per cent together with the distance $\bar{d}$ between the histograms in 
per cent are presented in Table 2. It can be observed that all these characteristics change with the introduction and the growth of a crack.

It should be noted though that a crack with length less than $5 \%$ of the plate radius causes rather small changes in all the observed parameters $\bar{d}, \sigma$ and $s$. This suggests that such cracks with length $<25 \mathrm{~mm}$ will be difficult to detect on the basis of these parameters.

The next results correspond to the case of distributed damage, which may be caused by corrosion, erosion or multiple cracks for the case of brittle materials, when eventually the cracks cover a whole area of the structure. The damaged areas are shown on Figure 1. We consider two cases- central damage (A) when the centre of damaged zone is in the plate centre and an end damage (B) when the damaged zone is close to the plate end. In both cases the damaged area covers a small square $(50 \mathrm{~mm}$ by $50 \mathrm{~mm}$ ) of the plate surface. Damage is introduced by a certain stiffness reduction in the whole area. Different damage levels are introduced by different stiffness reductions - between $10 \%$ and $50 \%$. The same parameters as for the case of crack are tracked with damage. Figures 3 and 4 presents the histograms for the case of the non damaged plate as compared to those corresponding to different damage levels for damage case A. The same trends that were observed in the histograms for crack can be perceived in these histograms as well, namely the spread goes down with damage and the symmetry increases. So the biggest spread corresponds to the non damaged case, while the smallest one is the one corresponding to $50 \%$ stiffness reduction, which is the maximum damage considered here. And the most symmetric distributions are the ones corresponding to the highest level of damage $(50 \%$ stiffness reduction) while the least symmetric one is the one for the non damaged case. The results for the relative changes in the parameters $\bar{d}, \bar{\sigma}$ and $\bar{s}$ for the case A and case 
B damage are presented in Tables 3 and 4. The changes are bigger for the case of central damage A where all the inspected features change more with damage compared to the changes that take place for damage case B. It can be observed that all the suggested parameters $\bar{d}, \bar{\sigma}$ and $\bar{s}$ undergo sufficient changes with the distributed damage and stiffness reduction from $10 \%$ on can be detected on the basis of these parameters. It should be noted that $10 \%$ stiffness reduction for the case of end damage B causes much bigger changes in $\bar{\sigma}$ and $\bar{s}$ compared to the changes induced in $\bar{d}$, which are rather small to be used for damage detection.

\section{Noise sensitivity of the proposed method.}

To test the robustness and the noise sensitivity of the developed method and the damage features to noise, a number of vibration response vectors were generated by adding a certain level of random white noise to the FE generated acceleration response. Different noise levels were investigated, namely $1 \%, 3 \%$ and $5 \%$ noise. Each data set corresponding to certain noise level contained 1000 vibration response vectors in total, 100 of each type of damage included in the investigation (see Table 5.). Table 5 shows the number of correctly recognised (as damaged or non-damaged) cases. It can be seen from the table that in general the percentage of correctly recognised cases is rather high- not less than $89 \%$. The highest erroneously recognised cases corresponds to low damage levels- 5\% crack and 10\% stiffness reduction. This is because for low damage the response of the plate is quite close to the original response and the addition of noise makes even more undistinguishable from the undamaged response. It can be noticed that the noise level increases the miss-recognised cases - the highest percentage of erroneously classified as nondamaged cases (10-11\%) correspond to the 5\% noise level. But in general the method and the suggested damage indexes demonstrate quite good performance even with 
noise contaminated data. Thus the method should be applicable to real data which normally contains a certain level of noise. All the three suggested damage indexes demonstrate similar performance for noisy data there is no evidence that any of the indexes performs considerably better or worse than the rest. In conclusion it should be noted that any of the suggested damage indexes can be used quite confidently even in noisy environment.

\section{Some comments and deductions.}

This study deals with the problem for damage detection in a thin circular plate. Two types of damage are considered - crack and distributed damage located in the centre of the plate (damage A) and close to the end (damage B). The paper investigates first the sensitivity of the lower natural frequencies to both types of damage. The first several natural frequencies are easy to determine from experiment and should be always considered as a first resource for possible damage features. Another advantage of using the first several natural frequencies is that they can be determined when the structure is subjected to small amplitude vibrations, e.g. a tap. In this particular case the lower natural frequencies demonstrate observable changes for rather high level of damage for both damage types considered. Therefore they cannot be used as viable damage features.

This study also offers another approach for tracking the changes in the vibration response of the plate caused by damage, which is based on the plate's time domain response. This approach uses the distribution of points on the attractor of the dynamic system to extract damage sensitive features. The method suggested is quite generic since it is solely based on the measured structural vibration response. Therefore it permits development and application to different more complex structures, which are 
difficult to model precisely, as well as to real measured data. In this particular case it has been developed and applied to a FE model of a thin circular plate.

Three possible candidates for damage features are extracted after studying the joint histograms of state space variables for the non-damaged case and the cases of damage. The parameter which undergoes the biggest changes is the skewness of the distribution of points, while the distance between the histograms is the least changing one. According to our results central damage (A) can be detected more easily than a side defect (B) ( see Tables 3 and 4). All the three damage features suggested can be easily extracted from the measured vibration response and can be used to detect the presence of a crack and/or distributed damage at a rather low level. Therefore they all present viable damage features.

The noise sensitivity and the robustness of the method and the suggested damage features was tested using a numerical experiment adding certain levels of noise to the FE simulated vibration responses and using the number of correctly recognised ( as damaged or non-damaged cases). The success rate of the method using any of the suggested features was found to be around $90 \%$ and lower for the lower noise levels. Most of the miss-recognised cases corresponded to low damage and 5\% noise. On the basis of these results the method and the features offered were found to be robust enough to noise of up to about $5 \%$.

All the above conclusions are based on FE modelling and thus have to be experimentally confirmed. 


\section{References}

1. Gorman D.G., Trendafilova I., Mulholland A.J., Horácek J., Vibration of a Coupled Plate/Fluid Interacting System and its Implication for Modal Analysis and Vibration Health Monitoring, 2006, Applied Mechanics and Materials 5-6, pp 323 330.

2. Gorman D.G., Trendafilova I., Mulholland A. and Horáček J.,Vibration analysis of a circular plate in interaction with an acoustic cavity leading to extraction of structural modal parameters, 2007, Journal of thin-walled structures (submitted).

3. Trendafilova I., Manoach E.,. Cartmell M.P,. Ostachowicz W.M, Zak A., An investigation on damage detection in aircraft panels using nonlinear time series analysis, 2007, Key Engineering Materials, vol.347, pp213-218.

4. Trendafilova I., Vibration-based damage detection in structures using time series analysis, 2006, Journal of Mechanical Engineering Science, Proceedings of the Institution of Mechanical Engineers Part C, Volume 220, Number 3, pp 261-272.

5. Todd M., J M Nichols, L M Pecora and L Virgin, Vibration-based Damage Assessment Utilizing State Space Geometry Changes: local Attractor Variance Ratio, 2001, Smart Mater. Struct. 10, pp 1000-1008.

6. Sohn H. and Farrar C., Damage diagnosis using time series analysis of vibration signals, 2001, Smart Mater. Struct. 10 pp 1-6.

7. Nichols J.M., Trickey S.T., Seaver M., Damage detection using multivariate recurrence quantification analysis, 2006, Mechanical Systems and Signal Processing 20, pp 421-437. 
8. Moniz L., Nichols J.M., Nichols C.J., Seaver M., Trickey S.T., Todd M.D., Pecora L.M., Virgin L.N., A multivariate, attractor-based approach to structural health monitoring, 2005, Journal of Sound and Vibration 283, pp 295-310.

9. Abarbanel H. Analysis of Observed Chaotic Data, 1996 (Springer Verl.)

10. Krantz H. and Schreiber T., Nonlinear time series analysis, 1997 (Cambridge University Press)

11. Fraser A.M. and Swinney H.L, Independent coordinates for strange attractors, 1986, Phys. Rev A, pp 1134-1140

12. Eckmann J-P. and D. Ruelle, Ergodic Theory of Chaos and strange attractors, 1985, Reviews of Modern Physics, vol 57, No 3, pp 617-655.

13. Mardia, K.V.: Measures of multivariate skewness and kurtosis with applications. 1970, Biometrika 57, 519-530.

14. Lazarov B., Trendafilova I., An Investigation on Vibration-Based Damage Diagnosis in Thin Plates, 2004, Structural Health Monitoring 2004, Proc. IInd European Workshop, Munich, July 2004, pp 76-81.

15. Israr A, Cartmell M.P, Krawczuk M, Ostachowicz W.M, Manoach E., Trendafilova I., Shishkina E.V., Palacz M., On Approximate Analytical Solutions for Vibrations in Cracked Plates, 2006, Applied Mechanics and Materials 5-6, pp 315322.

16. Manoach. E. Dynamic large deflection analysis of elastic-plastic Mindlin circular plates,1994, International Journal of Non-Linear Mechanics, Vol. 29, pp. 723-735

17. Hutchinson, J. R, Response of a free circular plate to a central transverse load. 1988, J. Sound and Vibration, 123, pp. 129-143 
18. Trendafilova I. E. Manoach, Vibration Based Damage Detection in Plates by Using Time Series Analysis, 2008, Mechanical Systems and Signal Processing vol.22, pp 1092-1106.

19. Doebling S.W., C.R. Farrar and M.B. Prime, A Summary Review of VibrationBased Damage Identification Methods, The Shock and Vibration Digest, Vol. 30, No. 2, (1998), pp. 91-105. 


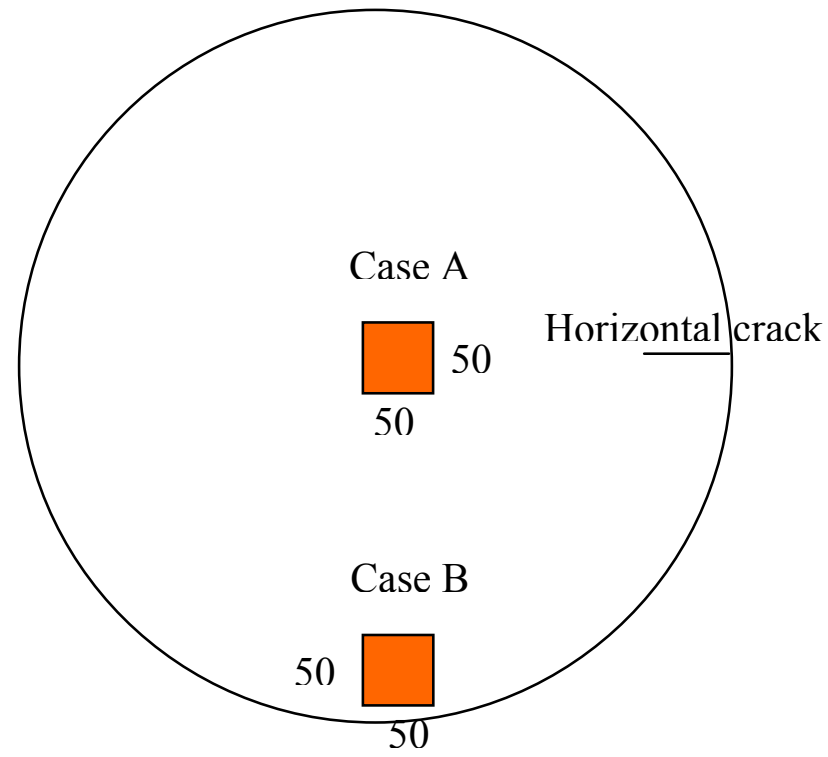

Figure 1. Plate with defects 

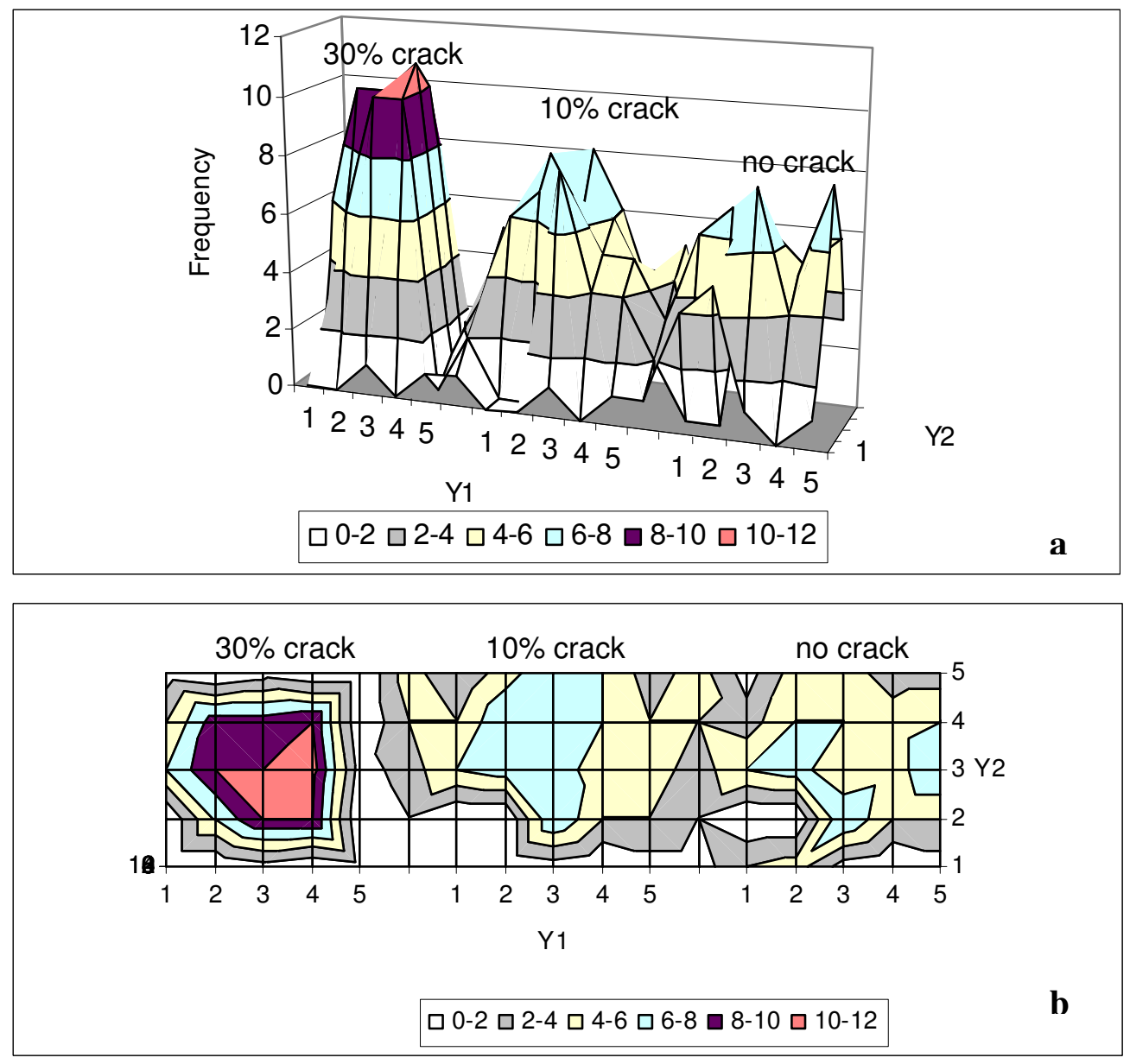

Figure 2. Histograms for crack as viewed from different perspectives (a and b) 


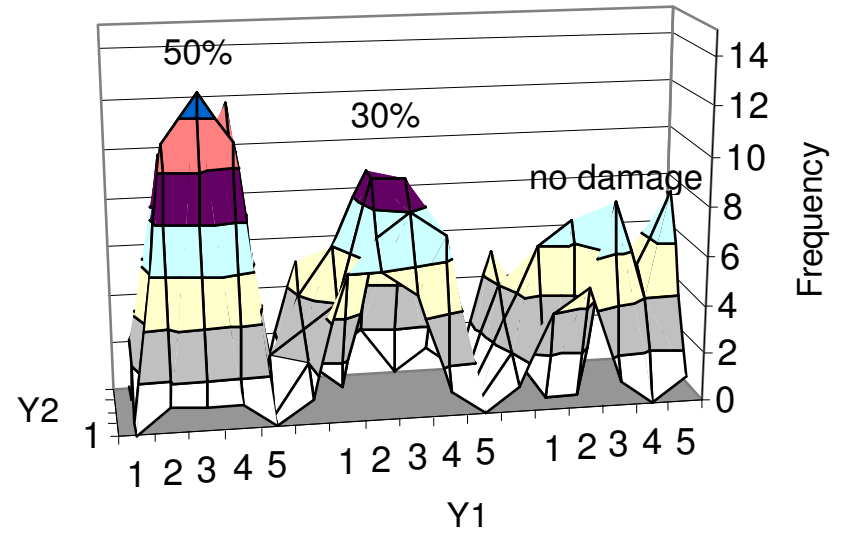

$\square$ 0-2 $\square$ 2-4 4-6 6-8 $\square$ 8-10 $\square$ 10-12 $\square$ 12-14

$\mathbf{a}$

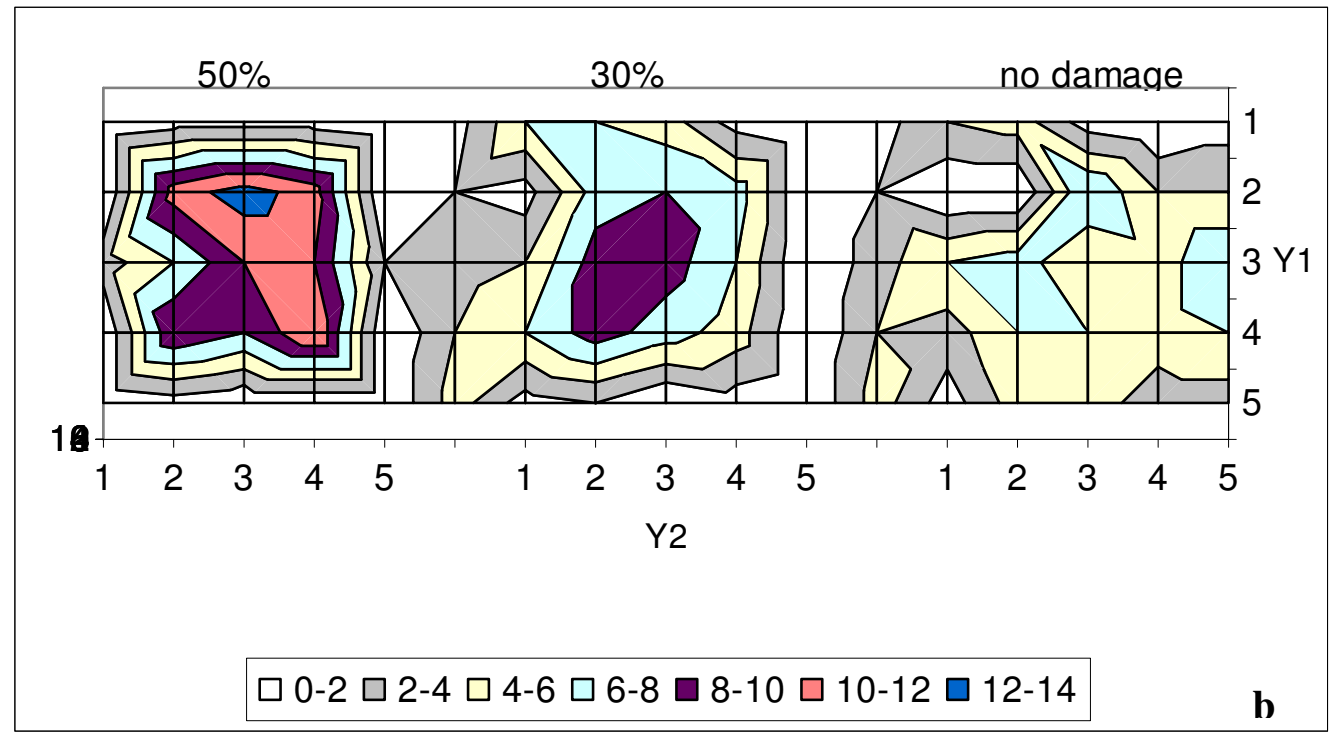

Figure 3. Histograms for distributed damage case $A$ as viewed from different perspectives ( $a$ and $b$ ) 


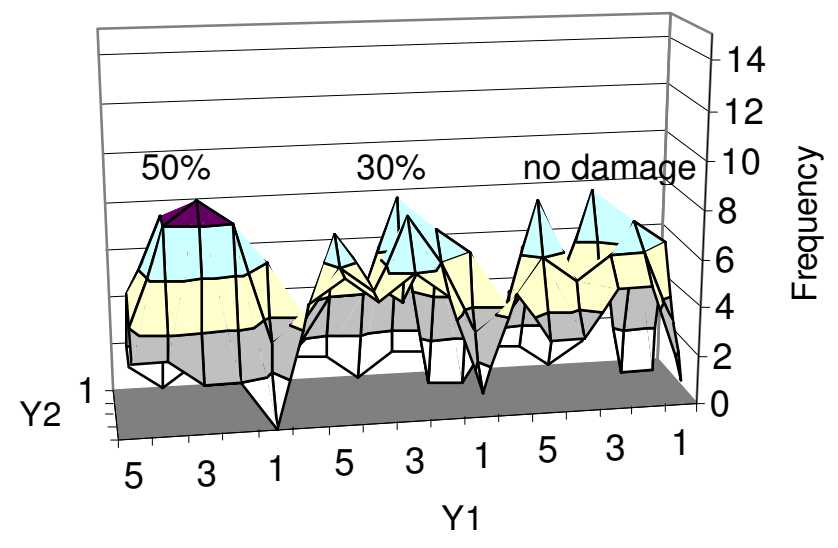

$\square$ 0-2 $\square$ 2-4 4-6 6-8 $\square$ 8-10 $\square$ 10-12 $\square$ 12-14

a

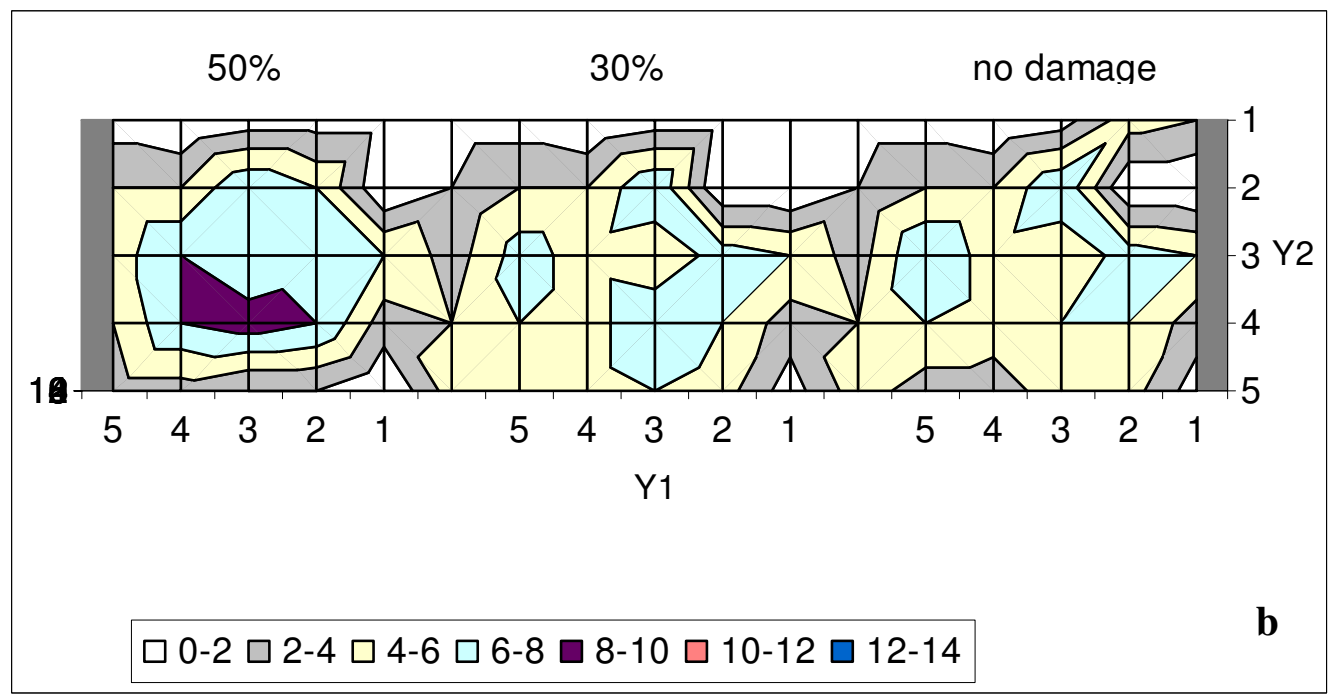

Figure 4. Histograms with distributed damage case $B$ as viewed from different perspectives (a and $b$ ) 


\begin{tabular}{|c|c|c|c|c|c|c|}
\hline defect & $\omega_{1}$ & $\omega_{2}$ & $\omega_{3}$ & $\omega_{4}$ & $\omega_{5}$ & $\omega_{6}$ \\
\hline $20 \%$ crack & 0.02 & 1.12 & 4.88 & 1.12 & 2.02 & 0.33 \\
\hline $30 \%$ crack & 0.20 & 2.11 & 5.55 & 3.05 & 2.90 & 1.05 \\
\hline $30 \%$ central defect & 1.02 & 3.09 & 5.01 & 3.12 & 2.10 & 0.50 \\
\hline $40 \%$ central defect & 1.33 & 3.40 & 6.02 & 3.30 & 2.22 & 0.55 \\
\hline $50 \%$ central defect & 1.55 & 4.11 & 6.77 & 3.99 & 3.01 & 1.02 \\
\hline $40 \%$ side defect & 0.91 & 4.98 & 4.01 & 2.11 & 1.33 & 0.89 \\
\hline $50 \%$ side defect & 1.50 & 5.56 & 4.50 & 3.16 & 1.55 & 1.12 \\
\hline
\end{tabular}

Table 1. Relative frequency changes in per cent with crack and distributed damage for central (A) and side (B) defect. 


\begin{tabular}{|c|c|c|c|c|c|}
\hline Crack size & $\begin{array}{c}\text { No } \\
\text { damage }\end{array}$ & $5 \%$ & $10 \%$ & $20 \%$ & $30 \%$ \\
\hline $\bar{d}$ & 0 & 4.55 & 9.12 & 20.10 & 26.12 \\
\hline$\sigma$ & 0 & 7.12 & 18.00 & 21.09 & 30.90 \\
\hline$s$ & 0 & 9.03 & 23.99 & 27.18 & 34.18 \\
\hline
\end{tabular}

Table 2. Average relative distance between histograms $\bar{d}$, relative variance change $\bar{\sigma}$ and relative skewness change $\bar{s}$ in per cent for the case of crack

\begin{tabular}{|c|c|c|c|c|c|c|}
\hline $\begin{array}{c}\text { Stiffness } \\
\text { reduction }\end{array}$ & $0 \%$ & $10 \%$ & $20 \%$ & $30 \%$ & $40 \%$ & $50 \%$ \\
\hline $\bar{d}$ & - & 5.01 & 14.02 & 25.20 & 30.12 & 32.01 \\
\hline $\bar{\sigma}$ & 0 & 5.15 & 40.09 & 44.12 & 46.53 & 50.14 \\
\hline $\bar{s}$ & 0 & 7.12 & 45.49 & 49.51 & 51.19 & 55.15 \\
\hline
\end{tabular}

Table 3. Average relative distance between histograms $\bar{d}$, relative changes in per cent of the multivariate variance $\bar{\sigma}$ and skewness $\bar{s}$ for the case of distributed damage- central defect (A)

\begin{tabular}{|c|c|c|c|c|c|c|}
\hline $\begin{array}{c}\text { Stiffness } \\
\text { reduction }\end{array}$ & $0 \%$ & $10 \%$ & $20 \%$ & $30 \%$ & $40 \%$ & $50 \%$ \\
\hline $\bar{d}$ & - & 2.01 & 6.11 & 12.09 & 18.05 & 31.12 \\
\hline $\bar{\sigma}$ & 0 & 6.46 & 9.16 & 21.33 & 26.54 & 40.36 \\
\hline $\bar{s}$ & 0 & 7.31 & 11.43 & 32.12 & 42.96 & 47.08 \\
\hline
\end{tabular}

Table 4. Average relative distance between histograms $\bar{d}$, relative changes in per cent of the multivariate variance $\bar{\sigma}$ and skewness $\bar{s}$ for the case of distributed damage- end defect (B) 


\begin{tabular}{|c|c|c|c|c|c|c|c|c|c|}
\hline & \multicolumn{3}{|c|}{$\begin{array}{l}\text { Distance between } \\
\text { histograms }\end{array}$} & \multirow[b]{2}{*}{$\begin{array}{c}1 \% \\
\text { noise }\end{array}$} & \multirow[b]{2}{*}{$\begin{array}{l}3 \% \\
\text { noise }\end{array}$} & \multirow[b]{2}{*}{$\begin{array}{c}5 \% \\
\text { noise }\end{array}$} & \multirow[b]{2}{*}{$\begin{array}{c}1 \% \\
\text { noise }\end{array}$} & \multirow[b]{2}{*}{$\begin{array}{c}3 \% \\
\text { noise }\end{array}$} & \multirow[b]{2}{*}{$\begin{array}{l}5 \% \\
\text { noise }\end{array}$} \\
\hline & $\begin{array}{c}1 \% \\
\text { noise }\end{array}$ & $\begin{array}{c}3 \% \\
\text { noise }\end{array}$ & $\begin{array}{c}5 \% \\
\text { noise }\end{array}$ & & & & & & \\
\hline defect & & & & & & & & & \\
\hline no defect & 99 & 98 & 96 & 98 & 97 & 90 & 100 & 98 & 96 \\
\hline $5 \%$ crack & 95 & 95 & 91 & 96 & 95 & 92 & 97 & 97 & 92 \\
\hline $\begin{array}{l}10 \% \\
\text { crack }\end{array}$ & 100 & 96 & 93 & 100 & 96 & 95 & 100 & 96 & 95 \\
\hline $\begin{array}{l}20 \% \\
\text { crack }\end{array}$ & 100 & 98 & 97 & 99 & 98 & 95 & 100 & 98 & 94 \\
\hline $\begin{array}{l}30 \% \\
\text { crack }\end{array}$ & 100 & 100 & 94 & 100 & 99 & 94 & 100 & 100 & 94 \\
\hline $\begin{array}{l}10 \% \\
\text { stiffness } \\
\text { reduction }\end{array}$ & 97 & 94 & 90 & 98 & 94 & 89 & 96 & 93 & 89 \\
\hline $\begin{array}{l}\quad 20 \% \\
\text { stiffness } \\
\text { reduction }\end{array}$ & 98 & 97 & 95 & 98 & 97 & 95 & 98 & 97 & 95 \\
\hline $\begin{array}{c}\quad 30 \% \\
\text { stiffness } \\
\text { reduction }\end{array}$ & 100 & 99 & 94 & 100 & 99 & 91 & 99 & 96 & 90 \\
\hline $\begin{array}{l}\qquad 40 \% \\
\text { stiffness } \\
\text { reduction }\end{array}$ & 100 & 100 & 98 & 100 & 99 & 99 & 100 & 100 & 96 \\
\hline $\begin{array}{l}\quad 50 \% \\
\text { stiffness } \\
\text { reduction }\end{array}$ & 100 & 99 & 95 & 99 & 98 & 95 & 100 & 98 & 95 \\
\hline
\end{tabular}

Table 5. Number/percentage of correctly recognized cases using the three different damage indexes for different noise levels and different defects 